\title{
Simulation on heat and mass transfer for a subcooled droplet falling freely in saturated steam by the lattice Boltzmann method
}

\author{
Qifang Luo ${ }^{1}$, Rui $\mathrm{Ma}^{2}$, Bo Dong ${ }^{1, *}$, Weizhong $\mathrm{Li}^{1}$, Jing Gong ${ }^{2}$ \\ 1. Key Laboratory of Ocean Energy Utilization and Energy Conservation of MOE, \\ School of Energy and Power Engineering, Dalian University of Technology, \\ Dalian, Liaoning, 116024, People's Republic of China
}

2. College of Mechanical and Transportation Engineering, China University of Petroleum, Beijing, 102249, China

*Address correspondence to Bo Dong, Key Laboratory of Ocean Energy Utilization and Energy Conservation of MOE, School of Energy and Power Engineering, Dalian University of Technology, No. 2 Linggong Road, Ganjingzi District, Dalian, Liaoning, 116024, People's Republic of China

E-mail: bodong@dlut.edu.cn

\begin{abstract}
:
In this paper, the growth and falling process of a single droplet in saturated steam is studied via the lattice Boltzmann method. The propagation of temperature field and flow field are directly simulated and the mechanism of heat and mass transfer is discussed. Moreover, the effect of relative velocity between droplet and steam on the growth and falling process of the droplet is also simulated. The results indicate the mean temperature of droplet with vertical velocity of steam is highest, while that with horizontal velocity is lowest. Furthermore, better condensation effect of droplet is obtained with smaller velocity of steam.
\end{abstract}

Key words: Lattice Boltzmann method; Subcooled droplet; Saturated steam; Heat and mass transfer 


\section{Nomenclature:}

$c$ lattice speed

$c_{s}$ lattice speed of sound

$C_{p l}$ specific heat at constant pressure

$D$ droplet diameter at initial stage

$d_{e}$ droplet equivalent diameter

$e_{\alpha}$ discrete velocity vector of LBM

Eo Eotvos number

$F_{\alpha}$ body force

$f_{\alpha}$ distribution function of continuity and momentum equations

$g$ gravitational acceleration

$g_{\alpha}$ distribution function of interface capturing equation

$h_{f g}$ latent heat

$h_{\alpha}$ distribution function of energy equation

Ja Jakob number

$n$ density

Oh Ohnesorge number

$P e$ Peclet number

$q$ constant parameter determined by $\tau_{\phi}$

$t$ time

$T$ temperature in fluid field

$T_{d}^{\prime}$ mean temperature of droplet

$T_{\infty}^{\prime}$ gas temperature far away from droplet $u$ macroscopic velocity of the fluid

$U_{T}$ droplet terminal falling velocity

$\delta$ discrete size

$\Gamma$ mobility coefficient

$\lambda_{l}$ thermal conductivity

$\mu_{l}$ kinetic viscosity of droplet

$\mu_{\phi}$ chemical potential

$\theta_{M}$ the mobility

$\rho$ density of fluid

$\tau_{n}$ dimensionless relaxation parameter for density

$\tau_{T}$ dimensionless relaxation parameter for temperature

$\tau_{\phi}$ dimensionless relaxation parameter for order

parameter

$\phi$ density difference ( order parameter )

$\sigma$ surface tension

$\omega$ weighting coefficient

\section{Subscripts/superscripts}

$d$ droplet

$e q$ equilibrium

$g$ gas

$l$ liquid

$\alpha$ discrete number 


\section{Introduction}

Multiphase flow with phase change and heat and mass transfer is a common phenomenon in the industrial applications. For example, in steam power stations, the dissolved oxygen in condensation water can cause oxidation corrosion to boiler, steam turbine, water supply system, pipeline and valve, so it is necessary to remove oxygen from the water as cleanly as possible, which can observably improve the security and reliability of the system $[1,2]$. The spray deaerator technique is a development tendency in modern power plant because it not only removes the oxygen but also reduces investment of equipments. The additional water sprayed by deaerator is on a subcooled state in the condenser. The temperature of the droplet must be heated to a saturated state, which corresponds to the steam partial pressure, so that the oxygen in the water can be removed. During the removal, the droplets produced in the deaerator can observably increase the heat and mass transfer, and moreover, the motion of the droplets can also enhance the heat transfer markedly. Therefore, it is necessary to understand the fundamental heat and mass transfer mechanism of a subcooled droplet in the saturated steam. Actually, the motion of subcooled droplets in saturated steam is a complicated process which refers to the phase change due to the temperature difference between the droplets and steam, as a result, many studies have been conducted.

As for the experimental study, Ford and Lekic [3] carried out experiments with single water droplets of three different diameters and with different initial subcooling of drops below the saturation temperature of steam to get the growth of drops. Mayinger and Chavez [4] performed an experiment with the aim of direct measurement of the growth of subcooled spray droplets in a pure saturated steam using a pulsed laser holography. In the work of Yang et al. [5], the growth of a condensing water droplet of size millimeters in steam flow is experimentally measured using a CCD camera. The growth rate of the droplet is found to increase with the steam flowrate. Hijikata et al. [6] conducted an experiment of condensation of steam on single droplets of $2.0 \mathrm{~mm}$, employing refrigerant R-113 and methanol as working fluids to utilize the subcooled state of the condensed liquid for enhancing a high performance condenser. Heat transfer of direct contact condensation to a droplet is rate-controlled by the heat transfer in the droplet. Experimental results show that heat transfer coefficient is about ten times higher than that of the solid sphere and about four times higher than the theoretical value, considering the circulate motion in the spherical droplet. Celata et al. [7] carried out an experiment with the aim of investigating the influence of droplet diameter and velocity, as well as the thermodynamic conditions of steam and water, on the heat transfer from saturated steam to subcooled water droplets. In the work of $\mathrm{Lu}$ and Melton [8], fluorescence shift thermometry has been combined with droplet slicing imaging to capture images of the 
transient temperature field within a 500- $\mu m$-diameter decane droplet falling into $370-420^{\circ} \mathrm{C}$ nitrogen.

Although lots of experiments have been carried out, and useful data are available, theoretical and numerical studies are still necessary to reveal the fundamental heat and mass transfer mechanism of a subcooled droplet in the saturated steam. In the theoretical analyses, pure conduction model, which is based on the assumption that the process of vapor condensation could be treated as transient heat transfer to a solid sphere with negligible resistance at the interface, is widely used. Ford and Lekic [3] studied the growth of the droplet due to steam condensation, neglecting the thermal resistance on the steam side and considering the droplet as a rigid sphere, i.e. using practically a pure conduction model. And then they [9] extended the model to the case of a spray of subcooled liquid droplets produced by a commercial full-cone type nozzle. The influence of certain parameters (initial drop velocity, drop size, etc.) on the thermal utilization was analyzed, and the drop size is found to be the most important. Pasamehmetoglu and Nelson [10,11] proposed an approach similar to that described above, and applied it to droplets in a condensing medium under transient conditions of pressure. Under steady conditions, the difference from the Ford and Lekic [3] model consists of the introduction of: (a) an empirical correction coefficient to take into account the convective contribution inside the droplet; and (b) a limitation of the steam supply towards the droplet surface. Ohba et al. [12] proposed a model based on the internal circulation in the droplets. They obtained the velocity fields inside the droplet and on the steam side by solving the conservation equations with the finite differences method. Rose and Kintner [13] developed a model for oscillating droplets of organic liquids in water under the basic hypothesis of the breaking of streamlines due to the oscillations, which gives rise to the internal mixing of the droplet. For Reynolds numbers exceeding about 200, the droplet may oscillate between an oblate (or spherical) and a more oblate shape. Such oscillations lead to a stretch of the interfacial surface and to significant internal mixing, which greatly enhances the rate of mass transfer. Several models are proposed in the literature and reviewed by Carra and Morbidelli [14]. Such models are usually complicated but may be approximated by empirical ad hoc expressions ( Skelland and Wellek ) [15]. Lai [16] carried out a study primarily aimed at theoretically investigating the growth of a single spherical droplet at the center of a large spherical container or in an infinite domain due to condensation. The effect on the droplet growth due to the subcooling, the Gibbs-Thomson condition, and the density-difference induced convection were analyzed and discussed systematically. 
As for the numerical simulation, two different classes of methods have been developed in the past to deal with the multiphase flow: tracking methods which consist of the moving mesh, front tracking, boundary integral and particle schemes, and capturing methods which include continuum advection, volume tracking, level set and phase field method schemes [17]. Enright et al. [18] with their particle level set method and Sussman et al. [19] with their coupled level set and volume of fluid method (CLSVOF) improved mass conservation significantly which cannot be guaranteed in the original level set method. Davidson and Rudman [20] presented their VOF-based algorithm for the calculation of transport processes across deforming interfaces. But this algorithm does not consider volume changes due to phase change and it is limited to axisymmetric cases. Jung and Sato [21] employed a moving, unstructured mesh to conduct 3D direct numerical simulations of a high Schmidt number flow over a droplet. The mesh consisted of prisms at the interface to resolve the viscous boundary layer and tetrahedral cells in the remaining domain. Gibou et al. [22] presented a similar method based on level set and Ghost Fluid, as well. They preserved the discontinuity of all variables but the viscosity which is smoothed across the interface.

Recently, the lattice Boltzmann method (LBM) has been proved to be an effective tool to study fluid flow and heat transfer problems due to its merits. Several LBM models have been developed for multiphase and multi-component fluid flow. The earlier one was the color method of Rothman and Keller [23]. Later, the potential method of Shan and Chen [24], the free energy method of Swift et al. [25] and the method of He et al. [26] had been proposed successively. These models have not been applied in practical problems due to the limitation of small density ratio. It is obvious that the density ratio between droplets and steam is large. Until 2007, two representative LBM models for large density ratio were proposed. One is the immiscible incompressible two-phase model with large density ratio proposed by Inamuro et al. [27], and the other was proposed by Zheng et al. [28]. In Inamuro's model, the interface was tracked by applying a diffuse equation which is analogy to Cahn-Hilliard equation. Unlike Inamuro's technique, Zheng et al. [28] used the C-H equation to track and define the two-phase interface without the artificial disposal, which is closer to the Landau mean-field theory than other models in the physical background. It has been pointed out that there is some criticism about the Zheng's model since it is incompressible and that's why it doesn't contain some terms. More details can be found in works of Taehun Lee[29-32]. However, as long as results are good and can be compared with other models, that is not the critical. On that basis, Dong proposed a hybrid LBM model [33], which was able to calculate the heat and mass transfer in multiphase flows through combining Zheng's multiphase model and a 
thermal LBM model [34]. In Dong's hybrid model, a source term was added in corresponding $\mathrm{C}-\mathrm{H}$ equation to define phase-change and the thermal LBM equation was added as a source term to define the latent heat. Such treatment can make the interface be tracked automatically based on the change of the phase order parameter.

During the past decades, the two-phase lattice Boltzmann method (LBM) has been successfully applied to investigate droplet transport under isothermal conditions [24, 35]. For non-isothermal two-phase flow, Inamuro et al. [36] proposed a DDF thermal LBM which neglected compression work and viscous heat dissipation. Subsequently, Hazi and Markus [37] considered the compression work and extended Inamuro's model to simulate heterogeneous pool boiling. Recently, Gong and Cheng [38] proposed a new scheme for the force term and used the exact difference method [39] for incorporating the new force term scheme in the LB method in order to obtain more accurate and stable numerical simulation results for two-phase flow under non-isothermal conditions. Subsequently, they derived a new form of source term in the energy equation [40] and applied the improved DDF Lattice Boltzmann method to study periodic bubble nucleation, growth and departure from a heated surface in pool boiling [40,41]. Most recently, Liu and Cheng [42] applied this improved LB model [38, 40] to study steady 2D laminar film condensation on a vertical hydrophilic flat plate at constant wall temperature. Their LB simulations were found in good agreement with the classical Nusselt model [43] and existing boundary layer solutions [44]. Subsequently, they simulated the transient formation of a 3D condensing droplet at a subcooled spot on a vertical hydrophobic flat plate and its subsequent dynamic behavior [45]. In the past few years, the lattice Boltzmann studies for liquid-vapor phase-change process were mostly focused on bubble rising, growth, breakup, coalescence, and so on. Nevertheless, to the best of our knowledge, the research of LBM about the droplet behavior with phase change and evolution of temperature field during droplet falling has not been found out. Therefore, in this paper, the lattice Boltzmann multiphase model combining with a thermal model is used to simulate a subcooled droplet falling freely in both quiescent and flowing saturated vapor steam, respectively. The aim of the present study is to test the feasibility of the recombination LBM model for numerical simulations of removing the oxygen from working medium. The numerical results can be considered as a basic work or a reference for generalizing LBM in the practical application about removing the oxygen from working medium. The paper is organized as follows. First, the lattice Boltzmann multiphase model is summarized in section 2. Then, the falling process of a subcooled droplet in static saturated steam is simulated in section 3 . The effect of vapor velocity on the falling process of a subcooled droplet is further 
discussed in section 4, respectively. Finally, the conclusions are made in section 5.

\section{Numerical method}

(1) The distribution functions of the interface capturing equation and momentum equation

Generally, the fluid is assumed to be Newtonian, incompressible fluid. In the present study, a lattice Boltzmann model is used to solve the governing equations including $\mathrm{N}-\mathrm{S}$ equations and $\mathrm{C}-\mathrm{H}$ equation. The interface capturing equation is modeled by a convective $\mathrm{C}-\mathrm{H}$ equation and corresponding lattice Boltzmann equation follows as

$g_{\alpha}\left(\vec{x}+\overrightarrow{e_{\alpha}} \Delta t, t+\Delta t\right)-g_{\alpha}(\vec{x}, t)=(1-q)\left[g_{\alpha}\left(\vec{x}+\overrightarrow{e_{\alpha}} \Delta t, t\right)-g_{\alpha}(\vec{x}, t)\right]-\frac{1}{\tau_{\phi}}\left[g_{\alpha}(\vec{x}, t)-g_{\alpha}^{e q}(\vec{x}, t)\right]$

where $\mathrm{q}$ is a constant parameter which is related to the dimensionless relaxation parameter by $q=\frac{1}{\tau_{\phi}+0.5}$

The distribution function of continuity and momentum equations can be described by

$$
f_{\alpha}\left(\vec{x}+\overrightarrow{e_{\alpha}} \Delta t, t+\Delta t\right)-f_{\alpha}(\vec{x}, t)=\Omega_{\alpha},
$$

with

$$
\Omega_{\alpha}=\frac{1}{\tau_{n}}\left[f_{\alpha}^{e q}(\vec{x}, t)-f_{\alpha}(\vec{x}, t)\right]+\left(1-\frac{1}{2 \tau_{n}}\right) \frac{\omega_{\alpha}}{c_{s}^{2}}\left[\left(\overrightarrow{e_{\alpha}}-\vec{u}\right)+\frac{\left(\overrightarrow{e_{\alpha}} \cdot \vec{u}\right)}{c_{s}^{2}} \overrightarrow{e_{\alpha}}\right]\left(\mu_{\phi} \vec{\nabla} \phi+\vec{F}_{\alpha}\right) \delta t
$$

In two-dimension, D2Q9 model is used. The discrete velocities are given by

$$
\left(\overrightarrow{e_{0}}, \overrightarrow{e_{1}}, \overrightarrow{e_{2}}, \overrightarrow{e_{3}}, \overrightarrow{e_{4}}, \overrightarrow{e_{5}}, \overrightarrow{e_{6}}, \overrightarrow{e_{7}}, \overrightarrow{e_{8}}\right)=\left[\begin{array}{ccccccccc}
0 & 1 & 0 & -1 & 0 & 1 & -1 & -1 & 1 \\
0 & 0 & 1 & 0 & -1 & 1 & 1 & -1 & -1
\end{array}\right] \cdot c
$$

where $c$ is the lattice speed. The equilibrium distribution functions are defined as follows:

$$
g_{\alpha}^{e q}=\omega_{\alpha}\left(B_{\alpha}+\frac{3 \phi}{q} \overrightarrow{e_{\alpha}} \cdot \vec{u}\right)
$$

where $B_{0}=\frac{9}{4} \phi-\frac{15}{4} \Gamma \mu_{\phi}, B_{\alpha}=3 \Gamma \mu_{\phi}(\alpha \neq 0) . \Gamma$ is used to control the mobility, and $n, \phi$ are defined as: $n=\frac{\rho_{A}+\rho_{B}}{2}, \phi=\frac{\rho_{A}-\rho_{B}}{2}, \rho_{A}$ and $\rho_{B}$ stand for the density of fluid A and 
fluid B, respectively. Usually, the order parameters $\phi$ are positive value in liquid phase and negative value in vapor phase in order to distinguish the two-phase.

$$
f_{\alpha}^{e q}=\omega_{\alpha} A_{\alpha}+\omega_{\alpha} n\left(3 \overrightarrow{e_{\alpha}} \vec{u}-\frac{3}{2} \vec{u}^{2}+\frac{9}{2}\left(\overrightarrow{e_{\alpha}} \vec{u}\right)^{2}\right)
$$

where the coefficients are taken as

$$
A_{0}=\frac{9}{4} n-\frac{15\left(\phi \mu_{\phi}+\frac{1}{3} n\right)}{4}, A_{\alpha(\alpha=1, \ldots .,)}=3\left(\phi \mu_{\phi}+\frac{1}{3} n\right), \omega_{0}=\frac{4}{9}, \omega_{\alpha(\alpha=1, \ldots ., 4)}=\frac{1}{9}, \omega_{\alpha(\alpha=5, \ldots \ldots .8)}=\frac{1}{36}
$$

The order parameter $\phi$, density $n$ and velocity are calculated as follows:

$$
\phi=\sum_{\alpha} g_{\alpha} \quad n=\sum_{\alpha} f_{\alpha} \vec{u}=\frac{1}{n}\left[\sum_{\alpha} f_{\alpha} \overrightarrow{e_{\alpha}}+\frac{1}{2}\left(\mu_{\phi} \vec{\nabla} \phi+\vec{F}_{\alpha}\right)\right]
$$

The gradient vector of order parameter $\vec{\nabla} \phi$ is calculated by

$$
\vec{\nabla} \phi=\frac{3}{c \Delta t}\left\{\begin{array}{l}
\dot{i} \sum_{\alpha=1}^{8} w_{\alpha}\left(\overrightarrow{e_{\alpha}} \cdot \vec{i}\right) \phi\left(\vec{x}+\overrightarrow{e_{\alpha}} \Delta t\right) \\
\vec{j} \sum_{\alpha=1}^{8} w_{\alpha}\left(\overrightarrow{e_{\alpha}} \cdot \vec{j}\right) \phi\left(\vec{x}+\overrightarrow{e_{\alpha}} \Delta t\right)
\end{array}\right.
$$

\section{(2) LBM thermal model}

In this paper, the thermal model proposed by Inamuro et al. [27] is used. The corresponding LBM equation is written as follows:

$h_{\alpha}\left(\vec{x}+\overrightarrow{e_{\alpha}} \Delta t, t+\Delta t\right)-h_{\alpha}(\vec{x}, t)=-\frac{1}{\tau_{T}}\left[h_{\alpha}(\vec{x}, t)-h_{\alpha}^{e q}(\vec{x}, t)\right]$

where $\tau_{T}$ is the dimensionless relaxation parameter for temperature.

In D2Q9 model, the equilibrium distribution function is

$$
h_{\alpha}^{e q}(\vec{x}, t)=\omega_{\alpha} T\left(1+3 \overrightarrow{e_{\alpha}} \cdot \vec{u}\right)
$$


where $\mathrm{T}$ is the dimensionless temperature of flow field.

The macroscopic dimensionless temperature is calculated by

$$
T=\sum_{\alpha} h_{\alpha}
$$

\section{(3) Consideration of phase change processing}

In the landau mean-field theory [46], a continuous variable of order parameter is used to describe the phase change. Thus, Dong [33] proposed a hybrid LBM model, which the C-H equation can be extended by including a phase change term in the non-isothermal system. The phase change process can be identified by the variation of order parameter. Meanwhile, the phase change latent heat is also considered in the combined LBM thermal model.

In order to study the mechanism of the subcooled droplet falls in the saturated vapor, a subcooled droplet of volume $V_{d}^{\prime}$ is introduced into the saturated vapor in interval of $t^{\prime}$ to $t^{\prime}+\Delta t^{\prime}$, and the mass transferred at the interface during phase change is expressed as:

$$
\int_{V^{\prime}} \frac{\Delta m_{l}}{\Delta t^{\prime}} d V^{\prime}=\int_{V^{\prime}} \rho_{l} \frac{d V_{l}^{\prime}}{d t} d V^{\prime}=-\frac{1}{h_{f g}} \int_{S^{\prime}} \lambda_{l}\left(\frac{\partial T^{\prime}}{\partial x^{\prime}}\right)_{l} d S^{\prime}=-\frac{1}{h_{f g}} \int_{V^{\prime}} \lambda_{l}\left(\frac{\partial^{2} T^{\prime}}{\partial x^{\prime 2}}\right)_{l} d V^{\prime}
$$

where, $\rho_{l}$ is density of droplet, $T^{\prime}$ is temperature, $h_{f g}$ and $\lambda_{l}$ are the latent heat and thermal conductivity, respectively.

Based on the phase order parameter, the phase change is expressed as:

$\dot{\phi}=\frac{\Delta \phi}{\Delta t^{\prime}}=\frac{\left(\rho_{l}+\Delta m\right)-\left(\rho_{g}-\Delta m\right)}{2 \Delta t^{\prime}}-\frac{\rho_{l}-\rho_{g}}{2 \Delta t^{\prime}}=\frac{\Delta m}{\Delta t^{\prime}}$

The below normalized equations are substituted into the Eq. (12) and dimensionless is carried out.

$$
V_{d}=\frac{V_{d}^{\prime}}{V_{d 0}^{\prime}}, t=\frac{t^{\prime} U_{T}}{d_{e}}, T=\frac{T^{\prime}-T_{\infty}^{\prime}}{T_{d}^{\prime}-T_{\infty}^{\prime}}, x=\frac{x^{\prime}}{d_{e}}
$$

where, $V_{d 0}^{\prime}$ is the droplet volume at initial stage, $d_{e}$ is the equivalent diameter of the droplet, $U_{T}$ is terminal velocity of droplet. $T_{\infty}^{\prime}$ is the temperature of saturated vapor far 
away droplet. The dimensionless form of the Eq. (12) is rewritten as:

$\rho_{l} \frac{d V_{l}}{d t}=-\frac{\lambda_{l}\left(T_{d}^{\prime}-T_{\infty}^{\prime}\right)}{h_{f g} U_{T} d_{e}}\left(\frac{\partial^{2} T}{\partial x^{2}}\right)$

By introducing the dimensionless Jakob number and Peclet number $J a=\frac{1}{h_{f g}} C_{p l}\left(T_{d}^{\prime}-T_{\infty}^{\prime}\right)$ and $P e=\frac{\rho_{l} U_{T} d_{e} C_{p l}}{\lambda_{l}}$, the Eq. (14) can be expressed as

$$
\frac{d V_{l}}{d t}=-\frac{J a}{P e}\left(\frac{\partial^{2} T}{\partial x^{2}}\right)=\frac{\dot{\phi}}{\rho_{l}-\rho_{g}}
$$

Adding the source term of phase change into the Eq. (1), the new equation is expressed as follows:

$g_{\alpha}\left(\vec{x}+\overrightarrow{e_{\alpha}} \Delta t, t+\Delta t\right)-g_{\alpha}(\vec{x}, t)=(1-q)\left[g_{\alpha}\left(\vec{x}+\overrightarrow{e_{\alpha}} \Delta t, t\right)-g_{\alpha}(\vec{x}, t)\right]-\frac{1}{\tau_{\phi}}\left[g_{\alpha}(\vec{x}, t)-g_{\alpha}^{e q}(\vec{x}, t)\right]+\omega_{\alpha} \dot{\phi}(16)$

In the LBM thermal model, $P e=\frac{3}{\left(\tau_{T}-0.5\right) \delta}$. So the latent heat term $\frac{\dot{\phi}}{\left(\rho_{l}-\rho_{g}\right) J a}$ can be added into the Eq. (9) and the following equation is obtained as

$h_{\alpha}\left(\vec{x}+\overrightarrow{e_{\alpha}} \Delta t, t+\Delta t\right)-h_{\alpha}(\vec{x}, t)=-\frac{1}{\tau_{T}}\left[h_{\alpha}(\vec{x}, t)-h_{\alpha}^{e q}(\vec{x}, t)\right]+\omega_{\alpha} \frac{\dot{\phi}}{\left(\rho_{l}-\rho_{g}\right) J a}$

It should be noted that the equations (16) and (17) is only applied to lattice points which belong to the droplet, and as for lattice points in other areas, equations (1) and (9) are applied.

By using Taylor series expansion and the Chapman-Enskog expansion for equations (16) and (17), the macroscopic governing equations can be written as:

$$
\begin{aligned}
& \frac{\partial \phi}{\partial t}+\vec{\nabla} \cdot(\phi \vec{u})=\theta_{M} \nabla^{2} \mu_{\phi}-\left(\rho_{l}-\rho_{g}\right) \cdot \frac{J a}{P e}\left(\frac{\partial^{2} T}{\partial x^{2}}\right) \\
& \frac{\partial T}{\partial t}+u_{\alpha} \frac{\partial T}{\partial x_{\alpha}}=\delta \frac{1}{3}\left(\tau_{T}-\frac{1}{2}\right) \frac{\partial^{2} T}{\partial x_{\alpha}{ }^{2}}+\frac{\dot{\phi}}{\left(\rho_{l}-\rho_{g}\right) J a}
\end{aligned}
$$


When the external force is considered, the dimensionless number Eo, Oh are defined as follows:

$$
E o=\frac{g\left(\rho_{l}-\rho_{g}\right) D^{2}}{\sigma}, O h=\frac{\mu_{l}}{\sqrt{\rho_{l} D \sigma}}
$$

where $\mathrm{D}$ is the droplet diameter at initial stage, $\mu_{l}$ is viscosity of droplet, $\mathrm{g}$ is gravitational acceleration.

\section{Model Validation}

Here, a droplet falling process without phase change is simulated to verify the LBM model. First, the computational domain is divided into $200 \times 500$ lattice points. Fully developed boundary is applied at the all side walls.

The body force is gravitational force only:

$$
F_{\alpha}=\left(\rho_{l}-\rho_{g}\right) g
$$

The radius of droplet is 15 , and its initial location is set as $(100,460)$. The parameters are taken as $\rho_{l}=1000, \rho_{g}=200, \Gamma=800$.

The results are presented in terms of non-dimensional variables by Eq. (20). The effect of Eotvos number on deformation of the droplet is investigated. The Ohnesorge number is fix at 0.3 which corresponds to the kinematic viscosity of 0.1 . In the present study, the Eo number is varied from 6.5 to 43 by changing the gravity. The results are shown in Fig.1. At the lowest Eo number in Fig.1a, it is seen that the droplet deforms slightly and reaches a steady state with small deformations. In Fig.1b for Eo=11, the type of deformation is again small deformation, but the droplet is a little more deformed than that of the prior case. By increasing the Eo number to 22 in Fig.1c, the droplet first takes an elliptical shape with its back flattened. Gradually the back of the droplet is pushed and it stretches in the horizontal direction. Eventually, it becomes completely flat. In Fig.1d, Eo reaches to 43, and the initial stages of deformation are similar to the previous cases, but at final stages the droplet becomes slender at the poles and the shear breakup is seen, although the edges of the droplet have not detached yet. This behavior of droplet is expected. Because at higher Eo number the surface tension force is small and gravitational forces are dominant, and results in high deformation of the droplet. 

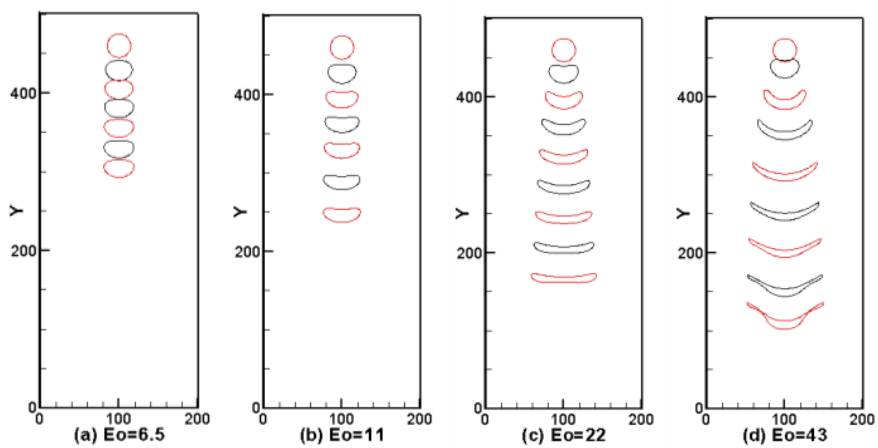

Fig.1 Deformation of a falling droplet at various Eo numbers for $\mathrm{Oh}=0.3$.
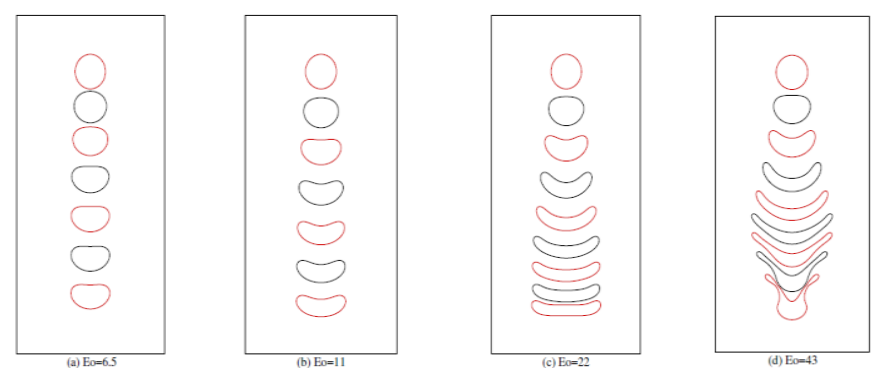

Fig.2 Snapshots of deformation of a falling droplet at various Eo numbers for $\mathrm{Oh}=0.3$ in the literature [48]

Fig.2 presents the snapshots of deformation of a falling drop under gravitational effect by Fakhari and Rahimian[48]. It is found that in terms of the droplet shape change, the numerical simulations are in good agreement with the literature. It should be noted that in the literature [48], the ranges of coordinates have not been provided (as shown in Fig.2), and in the present work, the deformation of droplet is snapshot in the whole computational domain. Considering that at larger Eo number, the droplet vertical velocity is bigger (as shown in Fig.3), thus the total falling distance of droplet is longer, the results are reasonable, so the model is reliable and can be used in the following studies.

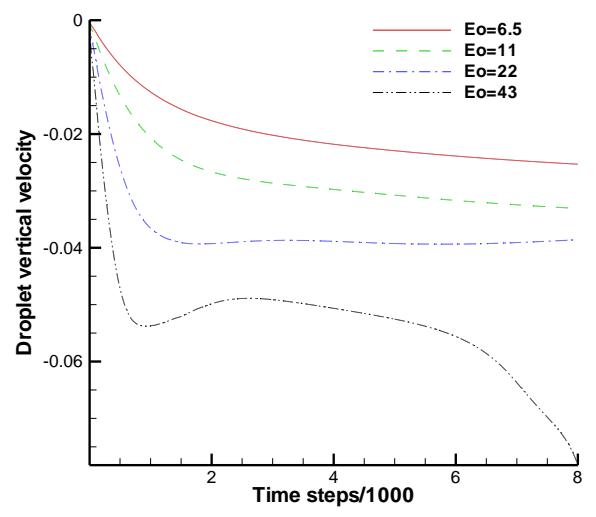

Fig.3 The vertical velocity of droplet at different Eo numbers 


\section{Simulation of a subcooled droplet falling in static saturated steam}

In this section, the falling process of a subcooled droplet in static saturated steam is simulated. The simulation is performed in a rectangular region. Fully developed boundary is employed [24] for boundaries. The vapor in computational domain is stationary. The initial temperature and force fields are calculated when the calculation time steps is bigger than 2000, in all the following simulations, in order to avoid the initial spontaneous diffusion.

Before the simulation is carried out, the effect of grid number in computational domain is studied. Fig.4 shows the effect of grid number in computational domain on the droplet shape. The corresponding grid sizes are $60 \times 550,120 \times 550,240 \times 550$ and $480 \times 550$, respectively. When $\mathrm{t}=0$, the static droplet fell down from the height of 515 under the effect of gravity. As shown in Fig.4(b), When $t=2600$, the height of droplets are 365, 347, 352 and 352, and that the diameter of droplets are 58,65, 55 and 55, respectively. And the droplet in the domain size of $60 \times 550$ is going to break. Therefore, when the simulation of droplet falling is conducted, the differences of deformation and location which are caused by the width of computational domain have to be considered. It has been pointed out that this kind of influence could be neglected when the width of domain is larger than four times of the droplet diameter [47]. Therefore, all simulations in this paper adopt computational domains that have a larger scale than four-times-droplet diameter to weaken the influence of width obviously.
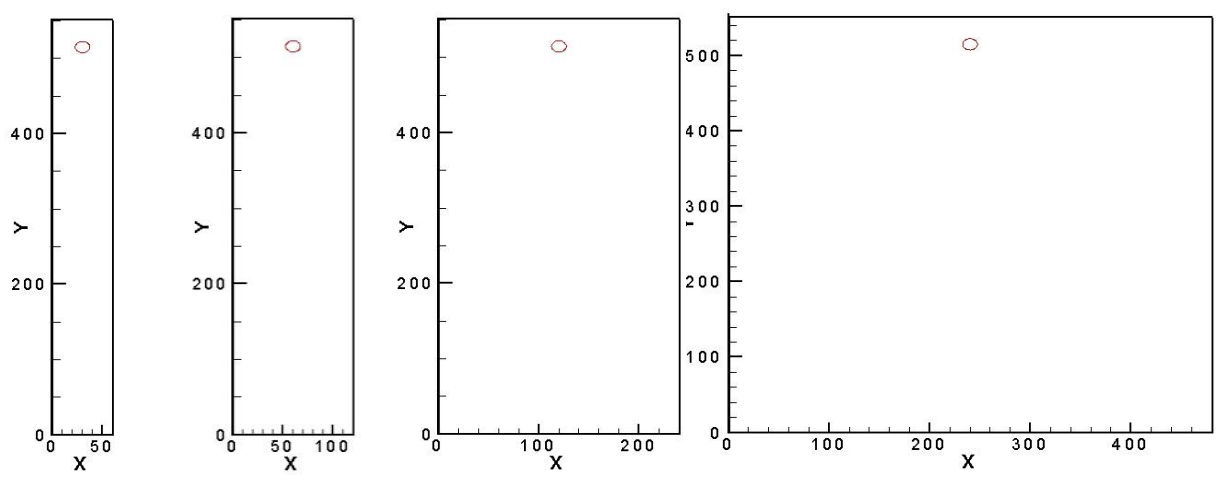

(a) when $\mathrm{t}=0$ time steps 

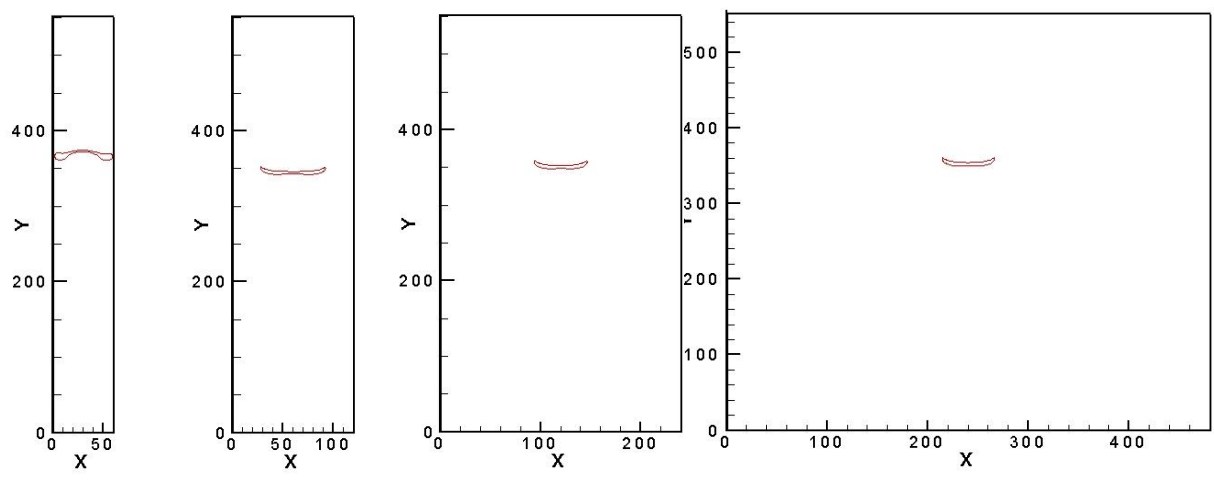

(b) when $\mathrm{t}=2600$ time steps

Fig.4 Effect of domain size in droplet shape from the left to the right, $60 \times 550,120 \times 550,240 \times 550$ and $480 \times 550)$

By using the above-mentioned model in section 2, the falling process of a droplet driven by gravity in static steam is simulated. Domain size is $240 \times 550$. The radius of droplet is 15 , and its initial location is set as $(120,500)$. Unless otherwise noted, the parameters of used are taken as $\rho_{l}=1000, \rho_{g}=1, \Gamma=800, J a=0.1488, P e=3000, \tau_{\phi}=1.5, \tau_{n}=0.8, \tau_{T}=0.503$. And initial conditions are listed as follows:

(1) droplet: $\quad E o=12.162162, O h=0.30015, u_{d}=0, v_{d}=0, T_{d}=0$;

(2) steam: $u_{g}=0, v_{g}=0, T_{g}=1$;

The shape evolution of a single droplet is shown in Fig.5. It can be seen that the droplet changes from circular oblate, then to kidney, and then to a long and flat wedge shape. Finally, the droplet stretches to both sides and it makes central of droplet taper and droplet breakage happen in this place under the influence of the gravity, drag force and interfacial tension. At initial stage, the interfacial tension plays a leading role in the droplet deformation due to small volume and velocity, and then during the falling process, the droplet grows due to steam condensation. As the time marched, the droplets became flatter due to the competition of gravity, drag force and interfacial tension. Fig.6 shows that the vertical velocity of droplet increases with the passage of time until the time steps of 11500. That is a combination of drag and gravity. At the time steps of 11500 , the drag on droplet which is a linear function of projected area of droplet on vertical direction is equal to the gravity. After that, the net force of droplet which shows as resistance increases with condensing and shape changing. 


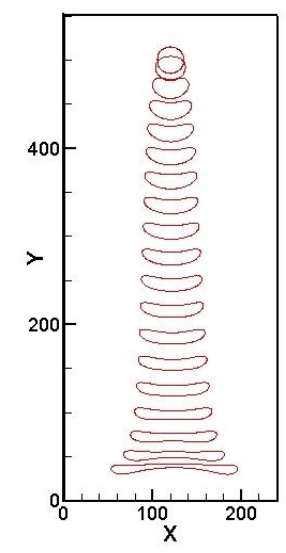

Fig.5 The shapes of single droplet at different time steps $(\Delta t=1000$ steps. $)$

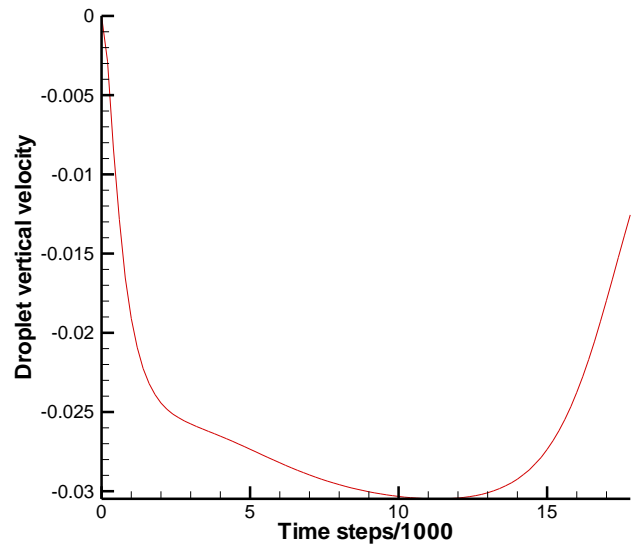

Fig.6 The vertical velocity of the falling droplet

The streamlines at different time steps are shown in Fig.7. At initial stage, two symmetrical semicircle vortexes which are caused by drag force are accompanied inside of droplet. With the process of time, drag force increases rapidly with increasing of relative velocity of two phases, meanwhile the shape change of droplet gets obviously. Then, there are two small symmetrical vortexes at the stern of droplet. And their effected area expands rapidly when the shape change of droplet gets more obviously. Contrarily, the vortexes in the droplet become more and more oblate until totally disappear at the end of stage.
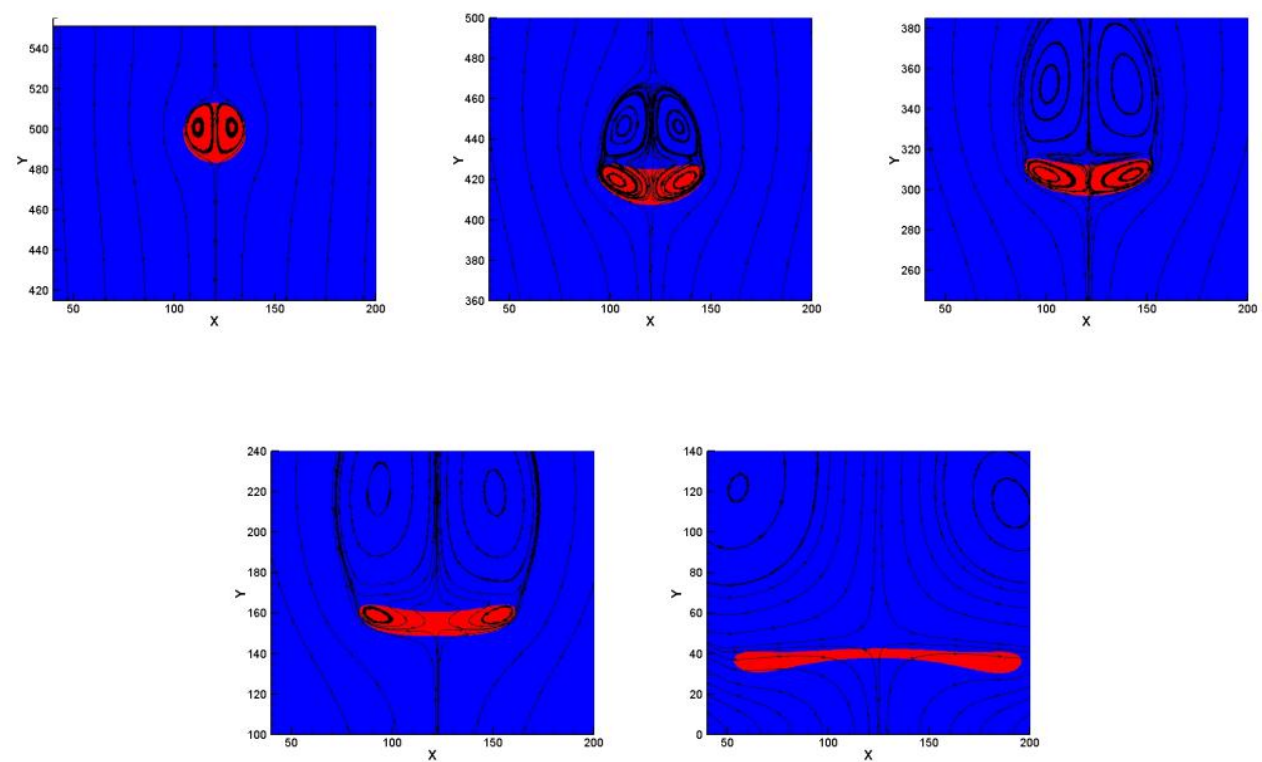

Fig.7 Streamlines at different time steps ( $t=500,4000,8000,13000,18000$ steps. $)$

The influence of steam condensation and movement of droplet on temperature field is presented in Fig.8. The black line is phase interface in which the order parameter equates to 0 . 
At initial time, a thermal boundary layer surrounding the droplet is generated in a very short time. At this stage, there is a large temperature gradient between the droplet and the steam, which leads to steam condensation and enhances the growth of droplet. But as the time goes, the temperature gradient decreases and the growth of droplet slows down due to the weaken rate of heat transfer. At the meantime, the combined action of drag force and gravity rolls non-saturated liquid to the bottom from interior of droplet, which enhances heat transfer between the non-saturated liquid and the steam. Compared with the scale of steam area, the size of droplet is so small that the existence of droplet has little influence on the steam temperature field.
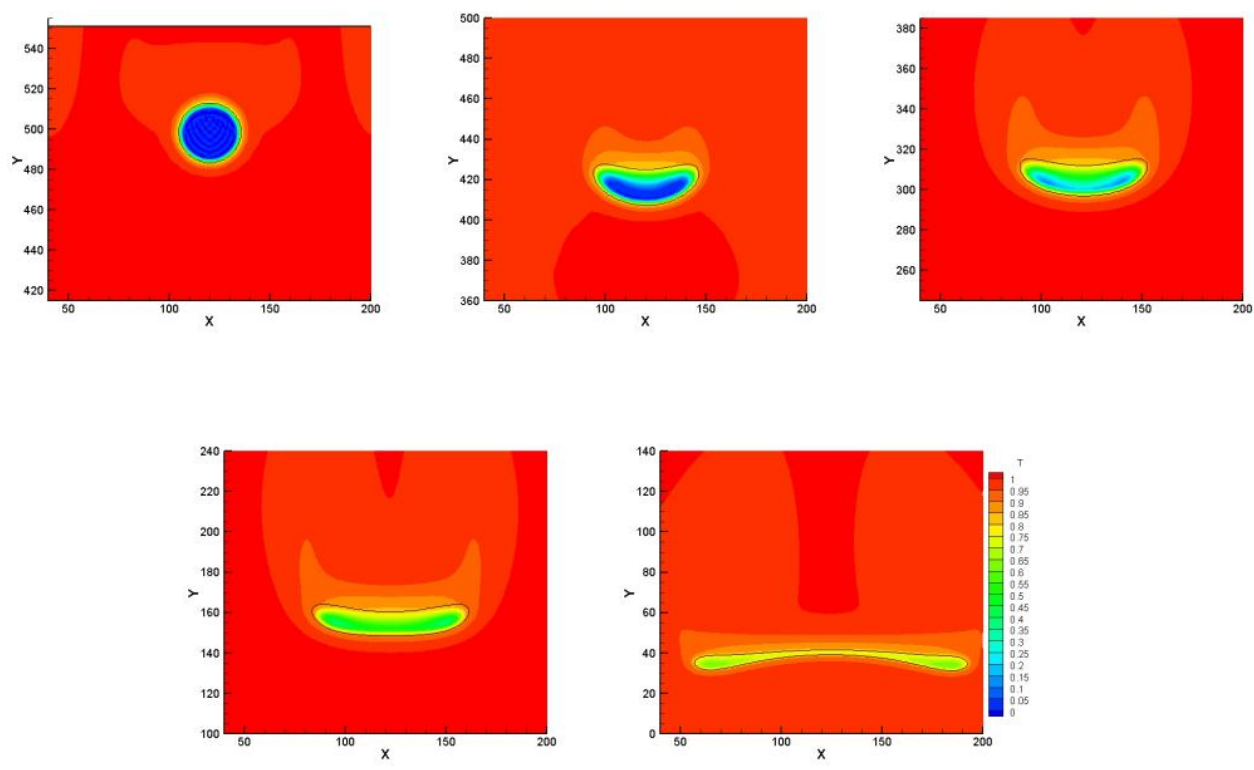

Fig.8 Propagation of temperature field ( $t=500,4000,8000,13000,18000$ steps. $)$

\section{Simulation of a droplet falling in saturated steam with velocity}

In this section, when a single droplet falls in a saturated steam flow, the influence of relative velocity between droplet and steam to heat transfer is studied by two cases.

Case one: The research domain is divided into $240 \times 550$ lattice nodes. The radius of droplet is 15 , and its initial location is set as $(120,500)$. Velocity boundary condition is applied at the bottom of domain, and fully developed boundary is used in other three boundaries. To prevent the upward movement of droplet, we set an initial velocity for droplet. And the initial conditions are listed as follows:

(1) Saturated steam: $u_{g}=0, v_{g}=0.01, T_{g}=1$. 
(2) Droplet: $E o=12.162162, O h=0.30015, u_{d}=0, v_{d}=-0.08, T_{d}=0$.

The detailed boundary conditions are listed as follows:

Bottom is inlet boundary: $u=u_{g}, v=v_{g}, T=T_{g}$;

Other boundary: $\frac{\partial u}{\partial x}=0, \frac{\partial v}{\partial x}=0, \frac{\partial T}{\partial x}=0$.

The shape evolution of a single droplet is shown in Fig.9. The shape evolutions of droplet in Fig.9 and Fig.6 are similar. The only difference is that the height of droplet in Fig.9 is higher than that in Fig.6 at the same time step, because the vertical steam has a hindrance to the droplet. Fig.10 shows the vertical velocity of droplet. The velocity of droplet decreases rapidly which is influenced by steam. But after that, it begins to increase slowly.

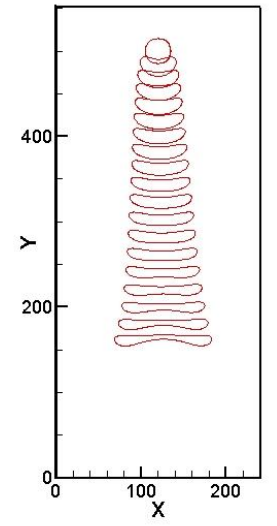

Fig.9 The shapes of single droplet at different time steps $(\Delta t=1000$ steps. $)$

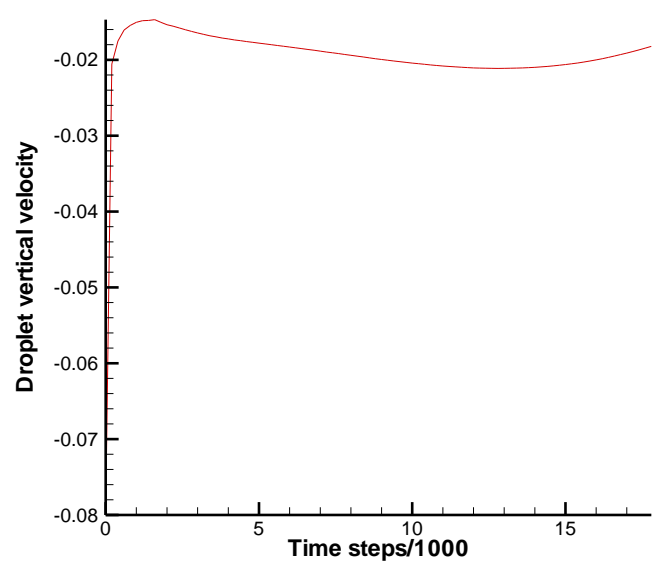

Fig.10 The vertical velocity of the falling droplet

By comparing Fig.11 with Fig.7, Fig.12 and Fig.8, it is found out that the evolutions of streamlines and temperature field are similar no matter the vertical velocity of steam is zero or not.
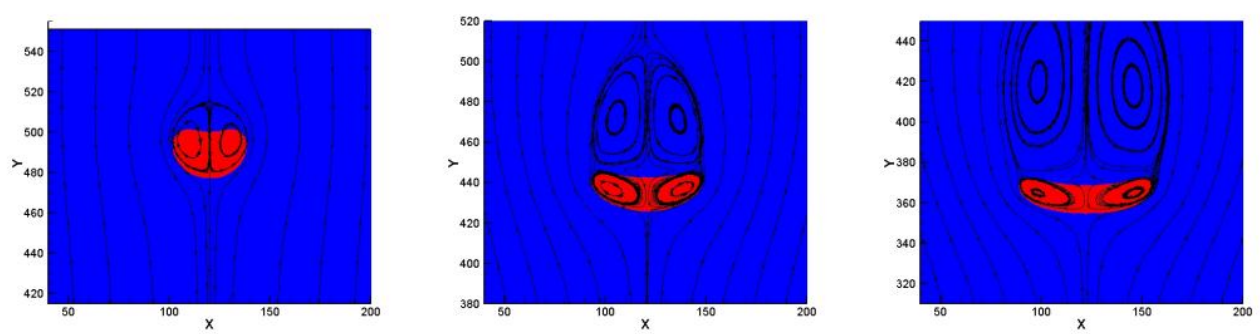

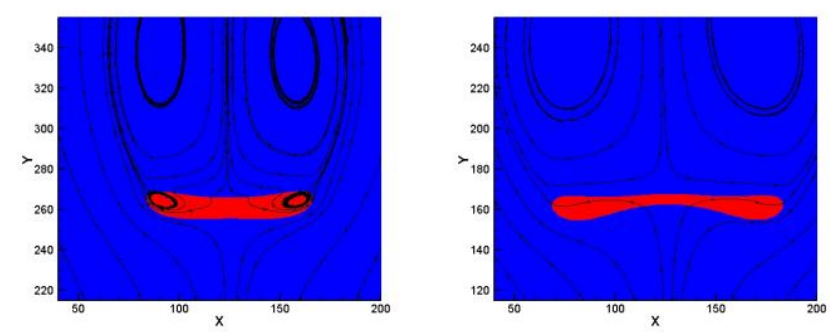

Fig.11 Streamlines at different time steps ( $t=500,4000,8000,13000,18000$ steps. $)$
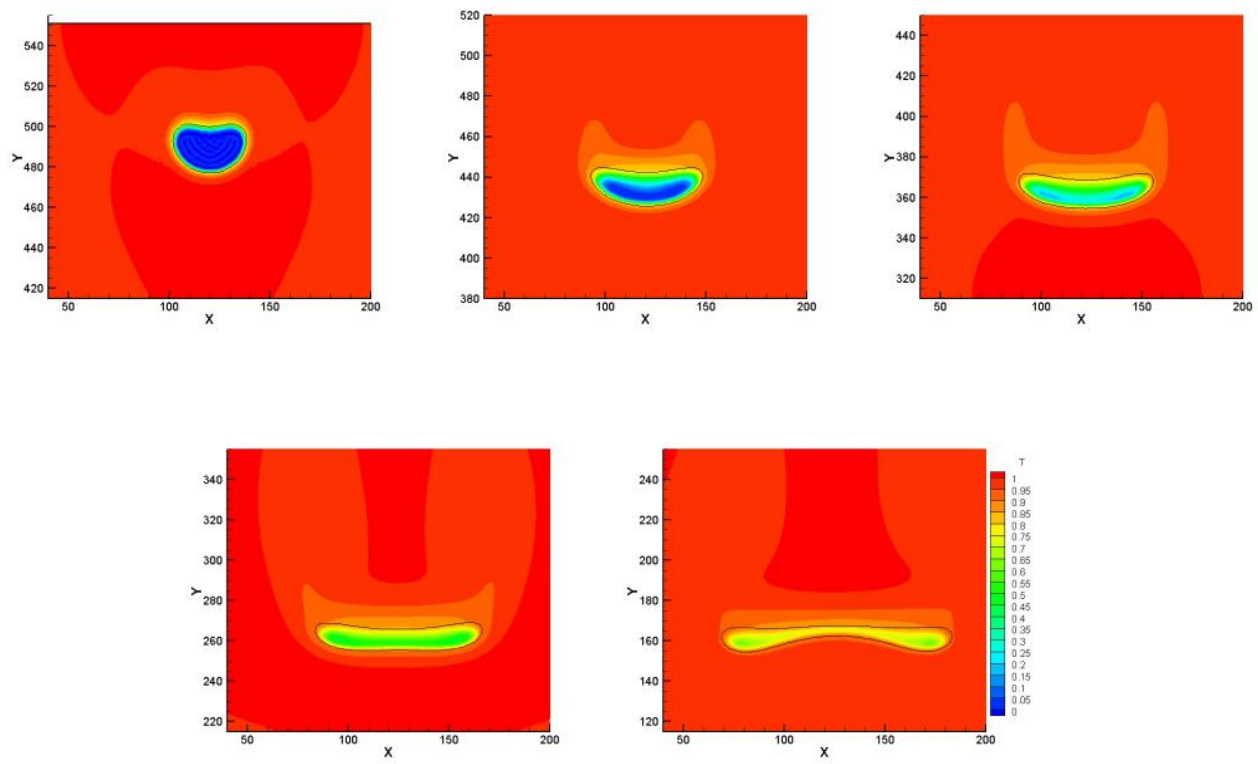

Fig.12 Propagation of droplet temperature field ( $t=500,4000,8000,13000,18000$ steps. )

Case two: The research domain is divided into $330 \times 500$ lattice nodes. The radius of droplet is 15 , and its initial location is set as $(50,470)$. Velocity boundary condition is applied at the left boundary of domain. Fully developed boundary is used in other three boundaries. The other initial conditions are listed as follows:

(1) Saturated steam: $u_{g}=0.01, v_{g}=0, T_{g}=1$.

(2) Droplet: $E o=12.162162, O h=0.30015, u_{d}=0, v_{d}=0, T_{d}=0$.

The detailed boundary conditions are listed as follows:

Left boundary is inlet boundary: $u=u_{g}, v=v_{g}, T=T_{g}$;

Other boundary: $\frac{\partial u}{\partial x}=0, \frac{\partial v}{\partial x}=0, \frac{\partial T}{\partial x}=0$. 
The evolution of the droplet shape at different time steps is shown in Fig.13. It is obviously that the droplet shape is asymmetric due to the existence of horizontal vapor velocity. Therefore, the droplet shape changes from circular to asymmetric semicircle, then to asymmetric kidney, finally to a long and flat asymmetric kidney shape. As shown in Fig.14, the evolution of vertical velocity of the falling droplet is similar to that in Fig.6, but it should be noted that when the time step is larger than 12000, the vertical velocity increases more rapidly instead of decreasing. The reason is that droplet diameter of this case which was influenced by horizontal velocity of vapor is smaller than that of Fig.6, so that the net force of droplet always acts as assistance. As the time steps pass, the relative balance of gravity and drag is broken, and then the vertical velocity of droplet increases more rapidly.

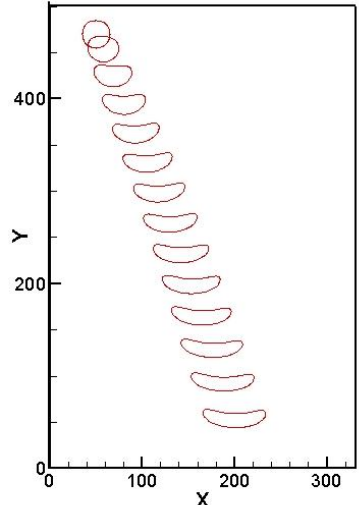

Fig.13 The evolution of the droplet shape at different time steps $(\Delta t=1000$ steps. $)$

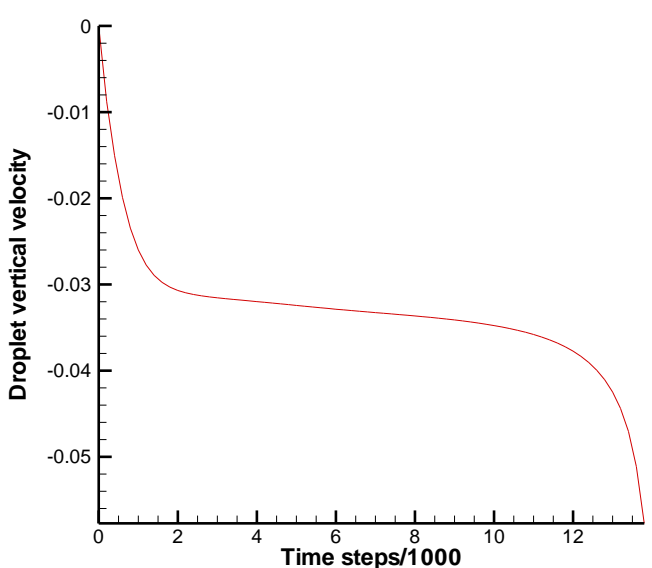

Fig.14 The vertical velocity of the falling droplet

The streamlines at different time steps is shown in Fig.15. At initial stage, two unsymmetrical semicircle vortexes named 1 for vortex on the left and named 2 for that on the right are accompanied inside of droplet, since horizontal steam makes net force unsymmetrical. And it can be seen that there is a difference in size between vortex 1 and 2. With the process of time, the shape change of droplet becomes obviously, meanwhile two unsymmetrical vertexes named 3 for the left one and named 4 for the right one appear in the back of droplet. With time passing, the effected area of vortexes 3 and 4 expands rapidly, but vertex 4 is always larger than vertex 3 in size. Then, vertex 4 disappears with vertex 3 becoming larger. Finally both of them disappear completely. Contrarily, the vortexes 1 and 2 become more and more oblate. The generation and extinction of four unsymmetrical vortexes inside and outside of droplet reflect the influence of steam horizontal velocity. As for temperature field, in the Fig.16, it is found out that the evolution of temperature is similar to the case that droplet falling in static saturated steam, namely, the relative velocity of droplet and steam do not 
have significant effect on the temperature field.
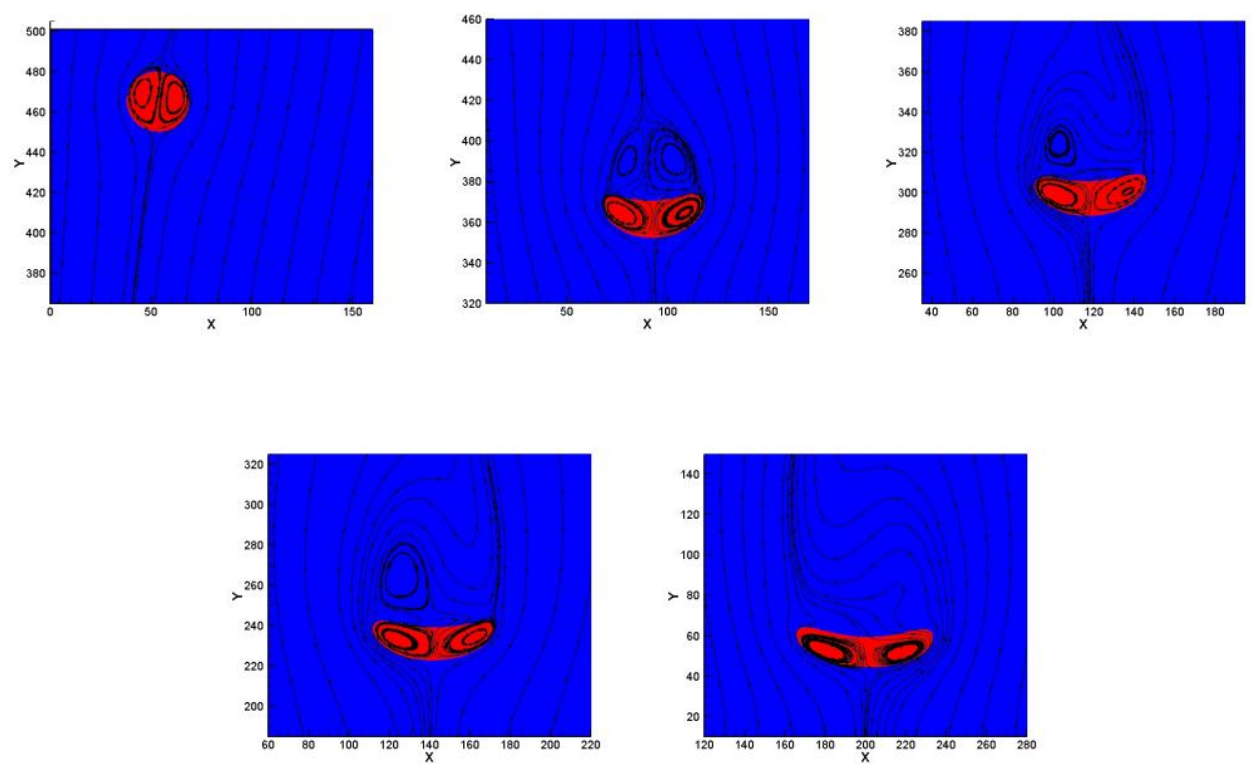

Fig.15 Streamlines at different time steps $(t=500,4000,6000,8000,13000$ steps. $)$
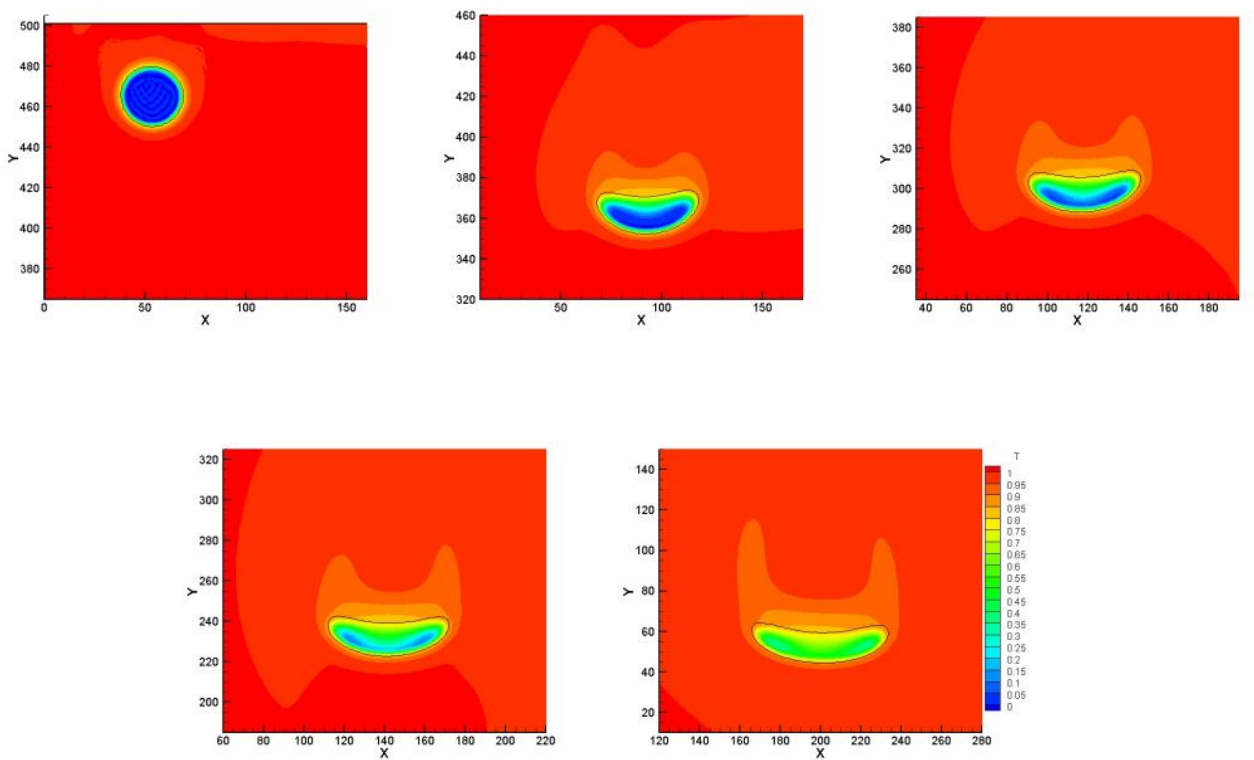

Fig.16 Propagation of droplet temperature field ( $t=500,4000,6000,8000,13000$ steps. )

Fig.17 shows the variations of droplet mean temperature and droplet equivalent radius with different steam velocities. It is found that when the horizontal and vertical velocities of steam are considered, the mean temperature of droplet with vertical velocity is a bit higher than that with others, while that with horizontal velocity is the lowest. However, the droplet equivalent radius with horizontal velocity becomes highest, which indicates that the droplet grows 
largest.
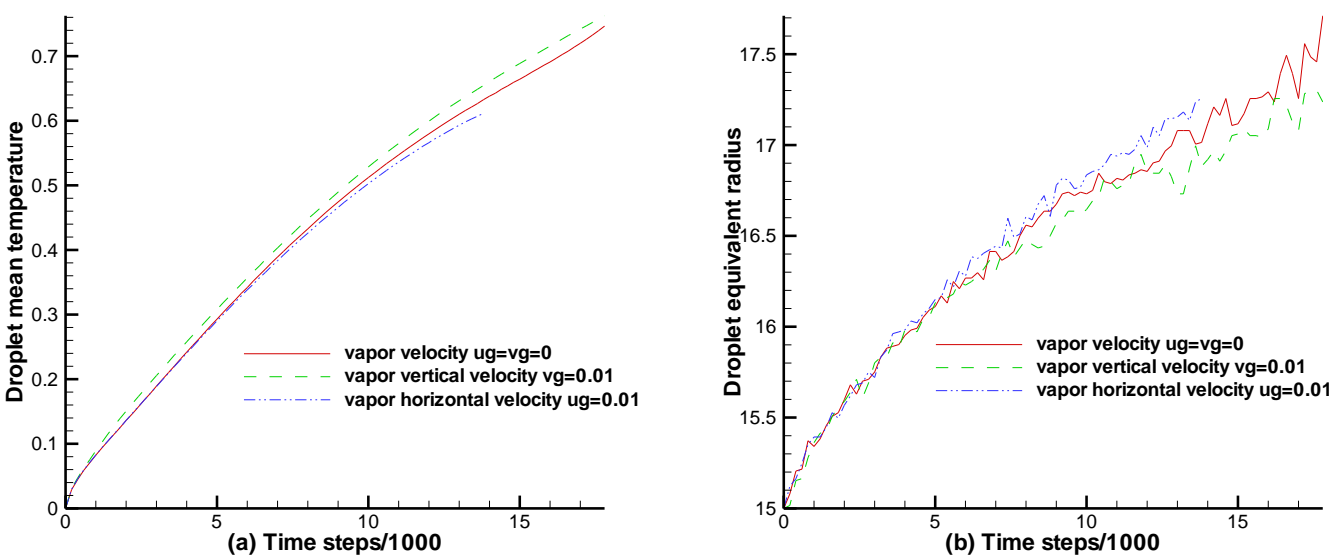

Fig.17 The variation of droplet mean temperature and equivalent radius with time steps for different vapor velocity

Fig.18 shows the influence of steam horizontal velocity on the subcooled droplet growth in the saturated steam. Considering the computing capacity of computer, the research domain is divided into $330 \times 500$ lattice nodes. The simulation is terminated when the droplet arrives at boundary of computational domain. As shown in Fig.18, the mean temperature of droplet increases with horizontal velocity of steam increased, while the equivalent radius of droplet decreases.
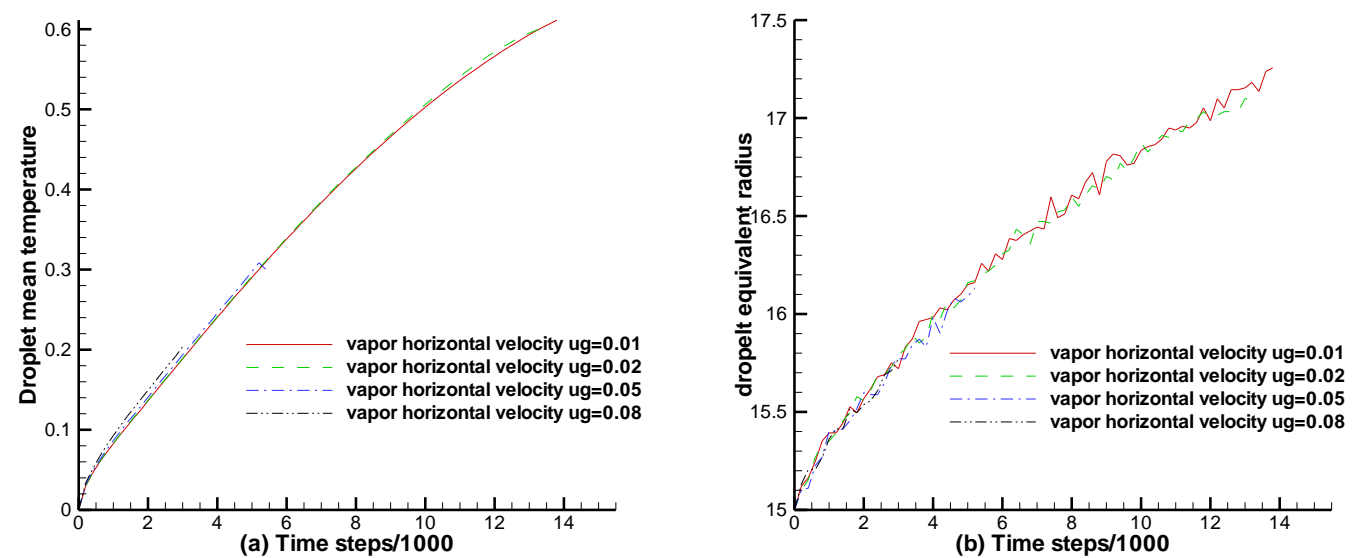

Fig.18 The variation of droplet mean temperature and equivalent radius with time steps for different vapor horizontal velocity

Fig.19 shows the influence of steam vertical velocity on the subcooled droplet growth in the saturated steam. It is found out that the droplet mean temperature does not change when the vertical velocity of steam varies at the beginning of the movement and medium term, as 
shown in Fig. 19(a). But at the late stage of falling, it becomes a bit higher when the vertical velocity of steam is greater. While as for the droplet equivalent radius, at the late stage, it becomes opposite.
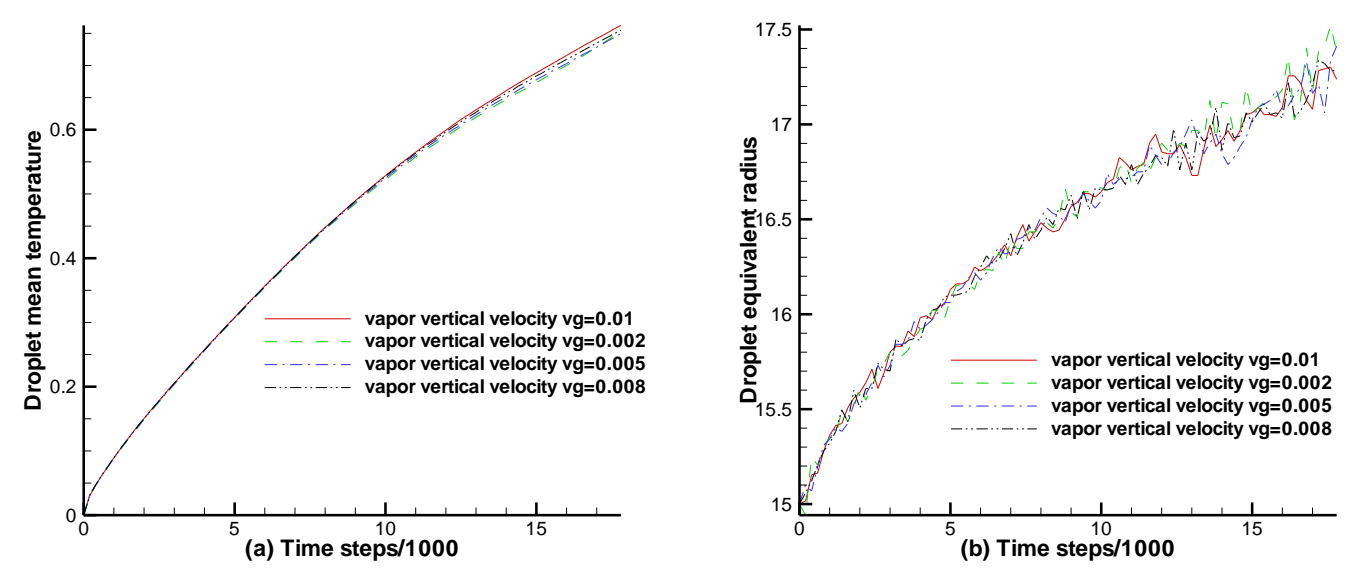

Fig.19 The variation of droplet mean temperature and equivalent radius with time steps for different vapor vertical velocity

\section{Conclusions}

In this paper, the condensation process of a subcooled droplet falling in saturated steam is simulated by the lattice Boltzmann method. First, a droplet falling process without phase change is simulated to verify the model by comparing with the results in the literature. Second, the grid sensitivity is checked to choose suitable lattice nodes for the following simulations. Then, the propagation of temperature field and flow field of droplet in static saturated steam are obtained. The results indicate that under the effect of gravity, drag force and interfacial tension, the droplet grows in the symmetrical manner due to the temperature difference between droplet and steam. Furthermore, the effect of steam velocity on the growth and falling process of droplet is studied. It is found that the mean temperature of droplet with vertical velocity of steam is highest, while that with horizontal velocity is lowest. However, as for the droplet equivalent radius, it is opposite. Furthermore, the droplet mean temperature increases with horizontal and vertical velocity of steam, while equivalent radius of droplet decreases. In other words, better condensation effect of droplet is obtained with smaller velocity of steam.

\section{Acknowledgement:}

This work is jointly sponsored by the National Science Foundation of China (51276030) and 
(51206017) and the Fundamental Research Funds for the Central Universities (DUT16QY03).

\section{References}

[1] Xu J., Compound condensate vacuum deaerating thermodynamic system of turbine unit. Electric Power Construction. 25(1): 9-11.

[2] Wang B., Thermal deaerating in the condenser. Power Station Auxiliary Equipment. 27(003): 5-7.

[3] FORD, J. D. \& LEKIC, A. 1973 Rate of growth of drops during condensation. Int. J. Heat Mass Transfer 16, 61-64.

[4] F. Mayinger, A. Chavez, Measurement of direct-contact condensation of pure saturated vapor on an injection spray by applying pulsed laser holography. Int. J. Heat Mass Transfer 35(3) (1992) 691-702.

[5]Yang Z, Ma X C, Duan Y Y. Internal flow and heat transfer of a condensing water droplet in steam flow. Chemical Engineering Science, 2013, 94: 54-59.

[6]HIJIKATA, K., MORI, Y. \& KAWAGUCHI, S. 1984 Direct contact condensation of vapor of falling cooled droplets. Int. J. Heat Mass Transfer 27, 1631-1640.

[7] CElatA, G. P., CUMO, M., D'ANNIBAle, F. \& FAREllo, G. E. Direct contact condensation of steam on droplets, Int. J. Multiphase Flow 17 (2) (1991) 191-211.

[8]Q. Z. Lu, and L. A. Melton, Measurement of transient temperature field within a falling droplet. AIAA Jouranl 38 (1) (2000).

[9] FORD, J. D. \& LEKIC, A. 1980 Direct contact condensation of vapor on a spray of subcooled liquid droplets. Int. J. Heat Mass Transfer 23, 1531-1537.

[10] PASAMEHMETOGLU, K. O. \& NELSON, R. A. 1987a Transient direct contact condensation on liquid droplets. Presented at the ASME-ANS-AIChE National Heat Transfer Conf., Pittsburgh, Pa.

[11]PASAMEHMETOGLU, K. O. \& NELSON, R. A. 1987b Direct contact condensation on liquid droplets during rapid depressurization, Part 1: quasi-steady solution. Presented at the ASME Winter A. Mtg, Boston, Mass.

[12]OHBA, K., KITADA, H. \& NISHIGUCHI, A. 1982 Direct contact condensation on a high speed spray jet of subcooled water. In Heat Transfer in Nuclear Reactor Safety (Edited by BANKOFF, S. G. \& AFGAN, N. H.). Hemisphere, Washington, D.C.

[13]ROSE, P. M. \& K1NTNER, R. C. 1966 Mass transfer from large oscillating drops. AIChE Jl 12, 530-534. 
[14]CARRA, S. \& MORmDELU, M. 1986 Transient mass transfer onto small particles and drops. In Handbook of Heat and Mass Transfer, Vol. 2 (Edited by CHER.EMISINOFF, N. P.), pp. 59-109. Gulf, Houston, Tex.

[15]SKELLAND, A. H. P. \& WELLEK, R. M. 1964 Resistance to mass transfer inside droplets. AIChEJ1 10, 491-496.

[16] Chun-Liang Lai, Gibbs-Thomson effect on droplet condensation. Transactions of the ASME, 121, AUGUST 1999.

[17] D.B. Kothe, Perspective on Eulerian finite volume methods for incompressible interfacial flows, in: Free Surface Flows, Springer-Verlag, 1998, pp. 267-331.

[18]D. Enright, R. Fedkiw, J. Ferziger, I. Mitchell, A hybrid particle level set method for improved interface capturing, Journal of Computational Physics 183 (2002) 83-116.

[19] M. Sussman, E.G. Puckett, A coupled level set and volume-of-fluid method for computing $3 \mathrm{~d}$ and axisymmetric incompressible two phase flows, Journal of Computational Physics 162 (2000) 301-337.

[20] M.R. Davidson, M. Rudman, Volume-of-fluid calculation of heat or mass transfer across deforming interfaces in two-fluid flow, Numerical Heat Transfer 41 (2002) 291-308.

[21] R.-T. Jung, T. Sato, Numerical simulation of high schmidt number flow over a droplet by using moving unstructured mesh, Journal of Computational Physics 203 (2005) 221-249.

[22] F. Gibou, L. Chen, D. Nguyen, S. Banerjee, A level set based sharp interface method for the multiphase incompressible Navier-Stokes equations with phase change, Journal of Computational Physics 222 (2007) 536-555.

[23]Rothman D H., Keller J M. Immiscible cellular-automaton fluid [J]. J. Statist. Phys. 1998, 52: $1119-1127$.

[24] Shan $X$ and Chen H. Lattice Boltzmann model for simulating flows with multiple phases and components [J]. Phys. Rev, 1993, 47(3): 1815-1819.

[25] Swift M R, Osborn W R and Yeomeans J M. Lattice Boltzmann simulation of non-ideal fluids [J]. Phys.Rev. Lett, 1995, 75: 830-833.

[26] He X, Chen S and Zhang R. A lattice Boltzmann scheme for incompressible multiphase flow and its application of Rayleigh-Taylor instability [J]. J.Comput.Phys, 1999, 152 (2): 642-663.

[27] Inamuro T, Ogata $T$, Tajima $S$ and Konishi N. A lattice Boltzmann method for incompressible two-phase flows with large density differences [J]. J. Comp. Phys, 2004, 198: 628-644.

[28] Zheng H W, Shu C and Chew Y T. A lattice Boltzmann for multiphase flows with large 
density ratio [J]. J. Comput. Phy, 2006, 218: 353-371.

[29] Lee, T. and Liu, L. (2010) Lattice Boltzmann Simulations of Micron-Scale Drop Impact on Dry Surfaces. Journal of Computational Physics, 229, 8045-8063.

[30] Amaya-Bower, L. and Lee, T. (2010) Single Bubble Rising Dynamics for Moderate Reynolds Number Using Lattice Boltzmann Method. Computers \& Fluids, 39, 1191-1207.

[31] Amaya-Bower, L. and Lee, T. (2011) Numerical Simulation of Single Bubble Rising in Vertical and Inclined Square Channel Using Lattice Boltzmann Method. Chemical Engineering Science, 66, 935-952.

[32] Lee, T. (2009) Effects of Incompressibility on the Elimination of Parasitic Currents in the Lattice Boltzmann Equation Method for Binary Fluids. Computers \& Mathematics with Applications, 58, 987-994.

[33] Dong, Z., Li, W., Song, Y., 2010. A numerical investigation of bubble growth on and departure from a superheated wall by lattice Boltzmann method. Int. J. Heat Mass. Trans 53, 4908-4916.

[34] Inamuro, T., Yoshino, M., Inoue, H., Mizuno, R., Ogino, F., 2002. A lattice Boltzmann method for a binary miscible fluid mixture and its application to a heat-transfer problem. J. Comput. Phys. 179, 201-215.

[35] L. Hao, P. Cheng, Lattice Boltzmann simulations of anisotropic permeabilities in carbon paper gas diffusion layers, J. Power Sources 186 (1) (2009) 104-114.

[36] T. Inamuro, M. Yoshino, H. Inoue, R. Mizuno, F. Ogino, A lattice Boltzmann method for a binary miscible fluid mixture and its application to a heattransfer problem, J. Comput. Phys. 179 (1) (2002) 201-215.

[37] G. Hazi, A. Markus, On the bubble departure diameter and release frequency based on numerical simulation results, Int. J. Heat Mass Transfer 52 (5) (2009) 1472-1480.

[38] S. Gong, P. Cheng, Numerical investigation of droplet motion and coalescence by an improved lattice Boltzmann model for phase transitions and multiphase flows, Comput. Fluids 53 (2011) 93-104.

[39] A.L. Kupershtokh, D.A. Medvedev, Lattice Boltzmann equation method in electrohydrodynamic problems, J. Electrostat. 64 (2006) 581-585.

[40] S. Gong, P. Cheng, A lattice Boltzmann method for simulation of liquid-vapor phase-change heat transfer, Int. J. Heat Mass Transfer 55 (2012) 4923-4927.

[41]S. Gong, P. Cheng, Lattice Boltzmann simulation of periodic bubble nucleation, growth and departure from a heated surface in pool boiling, Int. J. Heat Mass Transfer 64 (2013) $122-132$. 
[42] X.L. Liu, P. Cheng. Lattice Boltzmann simulation of steady laminar film condensation on a vertical hydrophilic subcooled flat plate, Int. J. Heat Mass Transfer 62 (2013) $507-514$.

[43] W. Nusselt, Die Oberflachenkondesation des Wasserdamffes the surface condensation of water, Zetrschr. Ver. Deutch. Ing. 60 (1916) 541-546.

[44]M.M. Chen, An analytical study of laminar film condensation. Part 1. Flat plates, J. Heat Transfer 83 (1961) 48.

[45]X.L. Liu, P. Cheng, Lattice Boltzmann simulation for dropwise condensation of vapor along vertical hydrophobic flat plates. Int. J. Heat Mass Transfer 64 (2013) 1041-1052.

[46]Chen, J.-h. and T. Lubensky (1976). Landau-Ginzburg mean-field theory for the nematic to smectic-C and nematic to smectic-A phase transitions. Physical Review A 14(3): 1202.

[47] Sun T, Li W, Yang S. Numerical simulation of bubble growth and departure during flow boiling period by lattice Boltzmann method [J]. International Journal of Heat and Fluid Flow,vol.44, 120-129, 2013.

[48] Fakhari, A. and M. H. Rahimian (2009). Simulation of falling droplet by the lattice Boltzmann method." Communications in Nonlinear Science and Numerical Simulation 14(7): 3046-3055.

\section{Appendix}

In this appendix, the Chapman-Enskog expansion process of macroscopic governing equations (1) and (2) have been presented.

$$
\begin{aligned}
\frac{\partial \phi}{\partial t}+\vec{\nabla} \cdot(\phi \vec{u}) & =\theta_{M} \nabla^{2} \mu_{\phi}-\left(\rho_{l}-\rho_{g}\right) \cdot \frac{J a}{P e}\left(\frac{\partial^{2} T}{\partial x^{2}}\right) \\
\frac{\partial T}{\partial t}+u_{\alpha} \frac{\partial T}{\partial x_{\alpha}} & =\delta \frac{1}{3}\left(\tau_{T}-\frac{1}{2}\right) \frac{\partial^{2} T}{\partial x_{\alpha}{ }^{2}}+\frac{\dot{\phi}}{\left(\rho_{l}-\rho_{g}\right) J a}
\end{aligned}
$$

First, the distribution function of interface capturing equation is written as follow:

$$
g_{\alpha}\left(\vec{x}+\overrightarrow{e_{\alpha}} \Delta t, t+\Delta t\right)-g_{\alpha}(\vec{x}, t)=(1-q)\left[g_{\alpha}\left(\vec{x}+\overrightarrow{e_{\alpha}} \Delta t, t\right)-g_{\alpha}(\vec{x}, t)\right]+\Omega_{\alpha}
$$

where, 


$$
\Omega_{\alpha}=-\frac{1}{\tau_{\phi}}\left[g_{\alpha}(\vec{x}, t)-g_{\alpha}^{e q}(\vec{x}, t)\right]+\omega_{\alpha} \dot{\phi}
$$

By using Taylor series expansion for equation (3), the distribution function has a second order accuracy and can be written as:

$$
\begin{aligned}
& \delta_{t}\left(\frac{\partial}{\partial t}+\vec{e}_{\alpha} \cdot \nabla\right) g_{\alpha}+\frac{\delta_{t}^{2}}{2}\left(\frac{\partial}{\partial t}+\vec{e}_{\alpha} \cdot \nabla\right)^{2} g_{\alpha} \\
& +(q-1)\left[\delta_{t}\left(\vec{e}_{\alpha} \cdot \nabla\right) g_{\alpha}+\frac{\delta_{t}^{2}}{2}\left(\vec{e}_{\alpha} \cdot \nabla\right)^{2} g_{\alpha}\right]+O\left(\delta_{t}^{3}\right)=\Omega_{\alpha}
\end{aligned}
$$

By using Chapman-Enskog expansions written as equations (6)-(9) for equation (5), the derivatives of distribution function are as equations (10)-(12):

$$
\begin{aligned}
& g_{\alpha} \approx g_{\alpha}{ }^{(0)}+\varepsilon g_{\alpha}{ }^{(1)}+\varepsilon^{2} g_{\alpha}{ }^{(2)} \\
& \frac{\partial}{\partial t} \approx \varepsilon \frac{\partial}{\partial t_{1}}+\varepsilon^{2} \frac{\partial}{\partial t_{2}} \\
& \nabla \approx \varepsilon \nabla_{1} \\
& \Omega \approx \Omega^{(0)}+\varepsilon \Omega^{(1)}+\varepsilon^{2} \Omega^{(2)}
\end{aligned}
$$

where $\varepsilon$ is an infinitesimal positive number.

$$
\begin{aligned}
O\left(\varepsilon^{0}\right): & g^{(0)}=g^{e q} \\
O\left(\varepsilon^{1}\right): & \varepsilon\left[\delta_{t}\left(\frac{\partial}{\partial t_{1}}+q \vec{e}_{\alpha} \cdot \nabla_{1}\right) g_{\alpha}{ }^{(0)}\right]=-\frac{\varepsilon}{\tau_{\phi}} g_{\alpha}^{(1)} \\
& \varepsilon^{2}\left\{\delta_{t} \frac{\partial}{\partial t_{2}} g_{\alpha}{ }^{(0)}+\delta_{t}\left(\frac{\partial}{\partial t_{1}}+q \vec{e}_{\alpha} \cdot \nabla_{1}\right) g_{\alpha}{ }^{(1)}\right. \\
O\left(\varepsilon^{2}\right): & \left.+\frac{1}{2} \delta_{t}^{2}\left[\left(\frac{\partial}{\partial t_{1}}+\vec{e}_{\alpha} \cdot \nabla_{1}\right)^{2}+(q-1)\left(\vec{e}_{\alpha} \cdot \nabla_{1}\right)^{2}\right] g_{\alpha}{ }^{(0)}\right\}=-\frac{\varepsilon^{2}}{\tau_{\phi}} g_{\alpha}{ }^{(2)}
\end{aligned}
$$

Simplify the equation (12) by using equation (11). Then the equation (12) can be written as follow: 


$$
\varepsilon^{2}\left\{\delta_{t} \frac{\partial}{\partial t_{2}}+\delta_{t}^{2}\left[\left(\frac{1}{2}-\tau_{\phi}\right) \frac{\partial^{2}}{\partial t_{1}}+\left(\frac{q}{2}-\tau_{\phi} q^{2}\right)\left(\vec{e}_{\alpha} \cdot \nabla_{1}\right)^{2}+\left(-2 \tau_{\phi} q+1\right) \frac{\partial}{\partial t_{1}} \vec{e}_{\alpha} \cdot \nabla_{1}\right]\right\} g_{\alpha}{ }^{(0)}=-\frac{\varepsilon^{2}}{\tau_{\phi}} g_{\alpha}{ }^{(2)}
$$

Then by summating the equations (11) and (13), we can get the equation (14).

$$
\begin{aligned}
& \delta_{t}\left(\varepsilon \frac{\partial}{\partial t_{1}}+\varepsilon q \vec{e}_{\alpha} \cdot \nabla_{1}+\varepsilon^{2} \frac{\partial}{\partial t_{2}}\right) g_{\alpha}{ }^{(0)}+\delta_{t}{ }^{2} \varepsilon^{2}\left[\left(\frac{1}{2}-\tau_{\phi}\right) \frac{\partial^{2}}{\partial t_{1}}+\left(-2 \tau_{\phi} q+1\right) \frac{\partial}{\partial t_{1}} \vec{e}_{\alpha} \cdot \nabla_{1}\right] g_{\alpha}{ }^{(0)} \\
& +\varepsilon^{2}\left(\frac{q}{2}-\tau_{\phi} q^{2}\right)\left(\vec{e}_{\alpha} \cdot \nabla_{1}\right)^{2} g_{\alpha}{ }^{(0)}=-\frac{\varepsilon g_{\alpha}{ }^{(1)}+\varepsilon^{2} g_{\alpha}{ }^{(2)}}{\tau_{\phi}}
\end{aligned}
$$

As a result, the distribution function of interface capturing equation can be written as:

$$
\begin{aligned}
& \delta_{t}\left(\frac{\partial}{\partial t}+q \vec{e}_{\alpha} \cdot \nabla\right) g_{\alpha}{ }^{(0)}+\delta_{t}{ }^{2}\left[\left(\frac{1}{2}-\tau_{\phi}\right) \frac{\partial^{2}}{\partial t_{1}}+\left(-2 \tau_{\phi} q+1\right) \frac{\partial}{\partial t_{1}} \vec{e}_{\alpha} \cdot \nabla_{1}\right] g_{\alpha}{ }^{(0)} \\
& +\delta_{t}^{2}\left(\frac{q}{2}-\tau_{\phi} q^{2}\right)\left(\vec{e}_{\alpha} \cdot \nabla_{1}\right)^{2} g_{\alpha}{ }^{(0)}+O\left(\delta_{t}{ }^{3}\right)=\Omega_{\alpha}
\end{aligned}
$$

After collating, the equation (15) can be written as follow:

$$
\begin{aligned}
& \delta_{t}\left(\frac{\partial}{\partial t}+q \vec{e}_{\alpha} \cdot \nabla\right) g_{\alpha}{ }^{(0)}+\delta_{t}^{2}\left\{\left[\left(\frac{1}{2}-\tau_{\phi}\right) \frac{\partial^{2}}{\partial t_{1}}+\left(\frac{1}{2}-\tau_{\phi}\right) q \frac{\partial}{\partial t_{1}} \vec{e}_{\alpha} \cdot \nabla_{1}\right] g_{\alpha}{ }^{(0)}\right. \\
& \left.+\left(\frac{2-q}{2}-\tau_{\phi} q\right) \frac{\partial}{\partial t_{1}}\left(\vec{e}_{\alpha} \cdot \nabla_{1}\right) g_{\alpha}{ }^{(0)}\right\}+\delta_{t}{ }^{2}\left(\frac{q}{2}-\tau_{\phi} q^{2}\right)\left(\vec{e}_{\alpha} \cdot \nabla_{1}\right)^{2} g_{\alpha}{ }^{(0)}+O\left(\delta_{t}{ }^{3}\right)=\Omega_{\alpha}
\end{aligned}
$$

The first and second order derivatives in equation (16) are high order infinitesimal, can be ignored. Meanwhile, the equilibrium distribution function of interface capturing equation must match conditions as follow:

$$
\sum_{\alpha} g_{\alpha}{ }^{(0)}=\phi, \sum_{\alpha} g_{\alpha}{ }^{(0)} \vec{e}_{\alpha i}=\frac{\phi}{q} \vec{u}, \sum_{\alpha} g_{\alpha}{ }^{(0)} \vec{e}_{\alpha i} \vec{e}_{\alpha j}=E_{i j}
$$

Then summate each item of equation (16) in each discrete direction, and the result can be written as:

$$
\frac{\partial \phi}{\partial t}+\nabla \cdot(\phi \vec{u})+\delta_{t}\left(\frac{2-q}{2 q}-\tau_{\phi}\right) \frac{\partial}{\partial t}[\nabla \cdot(\phi \vec{u})]+\delta_{t}\left(\frac{q}{2}-\tau_{\phi} q^{2}\right) \nabla^{2}\left(E_{i j}\right)-\dot{\phi}+O\left(\delta_{t}^{2}\right)=0
$$

In lattice Boltzmann model, the parameter $q$ is set to be $1 /\left(\tau_{\phi}+0.5\right)$. So equation (18) can be simplified as follow: 


$$
\frac{\partial \phi}{\partial t}+\nabla \cdot(\phi \vec{u})+\delta_{t}\left(\frac{q}{2}-\tau_{\phi} q^{2}\right) \nabla^{2}\left(E_{i j}\right)-\dot{\phi}+O\left(\delta_{t}^{2}\right)=0
$$

Where, momentum flux is defined as:

$$
E_{i j}=\Gamma \mu_{\varphi} \delta_{i j}
$$

The mobility is defined as:

$$
\theta_{M}=-\left(\frac{q}{2}-\tau_{\phi} q^{2}\right) \delta_{t} \Gamma
$$

Hence, we can achieve the macroscopic governing equation of interface capturing equation in the follow way:

$$
\frac{\partial \phi}{\partial t}+\nabla \cdot(\phi \vec{u})=\theta_{M} \nabla^{2} \mu_{\phi}-\left(\rho_{l}-\rho_{g}\right) \frac{J a}{P e}\left(\frac{\partial^{2} T}{\partial x^{2}}\right)
$$

Similarly, we can achieve the macroscopic governing equation (24) by using Taylor series expansion and Chapman-Enskog expansions for the energy equation (23).

$$
\begin{aligned}
& h_{\alpha}\left(\vec{x}+\overrightarrow{e_{\alpha}} \Delta t, t+\Delta t\right)-h_{\alpha}(\vec{x}, t)=-\frac{1}{\tau_{T}}\left[h_{\alpha}(\vec{x}, t)-h_{\alpha}^{e q}(\vec{x}, t)\right]+\omega_{\alpha} \frac{\dot{\phi}}{\left(\rho_{l}-\rho_{g}\right) J a} \\
& \frac{\partial T}{\partial t}+\nabla \cdot(T \vec{u})=\kappa \nabla^{2} T+\frac{\dot{\phi}}{\left(\rho_{l}-\rho_{g}\right) J a}
\end{aligned}
$$

where $\kappa=\frac{1}{3}\left(\tau_{T}-\frac{1}{2}\right) \delta_{t}$ 